\title{
Активность мобильных элементов - причина или следствие внутривидового гибридного дисгенеза у дрозофилы?
}

\author{
Л.П. Захаренко $\mathrm{D} \otimes$
}

\begin{abstract}
Аннотация: В статье приведены данные, противоречащие представлению о том, что внутривидовой гибридный дисгенез (ГД) у Drosophila melanogaster вызван массовым перемещением мобильных генетических элементов (МГЭ). По немногочисленным данным, скорость перемещения МГЭ при ГД не превышает один-два на геном за поколение. Активность мобильных элементов при ГД в большинстве работ оценена косвенно - по размерам яичника, фенотипическим проявлениям, уровню транскрипции транспозазы или активности генетических конструкций, искусственно внедренных в геном под активным промотором. МГЭ могут внедряться в разные гены, однако фенотипические симптомы ГД однотипны (при РМ ГД - недоразвитие яичников, при IR ГД - гибель эмбрионов) и для некоторых пар линий воспроизводятся со 100\% вероятностью. Обязательным условием РМ ГД является выращивание дисгенного потомства при повышенной температуре, однако перемещение МГЭ под влиянием температуры, если и проявляется, то только при тяжелом тепловом шоке, не совместимом с жизнедеятельностью дрозофилы. Степень выраженности симптомов РМ ГД слабо зависит от количества Р-элементов. Наличие полноразмерного $P$-элемента в геноме не гарантирует индукции ГД: подавляющая часть природных линий его содержит, но в качестве индуктора РМ ГД обычно используют референсную линию Harwich. Вклад МГЭ в ГД может быть не за счет массовых перемещений, а вследствие изменения конформации хроматина по соседству с его местом внедрения и, соответственно, смены регуляции активности прилежащих генов. Однако с учетом того что места внедрения МГЭ, как правило, непредсказуемы, а проявления ГД однотипны, требуется дальнейшее исследование характеристик референсных для ГД линий. Гибридный дисгенез значительно влияет на развитие, поэтому его невозможно объяснить только активацией транспозонов. Таким образом, истинную причину дисгенных событий еще предстоит выяснить с использованием современных методов.
\end{abstract}

Ключевые слова: гибридный дисгенез; скорость транспозиций; мобильные элементы; Drosophila melanogaster.

Благодарности: Работа поддержана бюджетным проектом № 0259-2021-0016. Автор благодарит И.К. Захарова за ценные замечания.

Для цитирования: Захаренко Л.П. Активность мобильных элементов - причина или следствие внутривидового гибридного дисгенеза у дрозофилы? Письма в Вавиловский журнал генетики и селекции. 2021;7(2):109-114. DOI 10.18699/LettersVJ2021-7-12

\section{Doubts about the involvement of transposable elements in the induction of hybrid dysgenesis in Drosophila melanogaster}

\section{L.P. ZakharenkoiD凶}

Abstract: This article reviews data contradicting to the hypothesis suggesting that the mass migration of transposable elements (TEs) is a cause of the intraspecific hybrid dysgenesis (HD) in Drosophila melanogaster. In the vast majority of reports, the activity of TEs at $\mathrm{HD}$ is estimated indirectly via the size of ovaries, via phenotypic manifestations, via the level of transposase transcription or the activity of genetic constructs artificially inserted into the genome under the active promoter. A small amount of available estimates of TEs migration at HD suggests the rate of one-two transpositions of definite TE per genome per generation. TEs can be introduced into different genes; however, the phenotypic symptoms of HD are the same (at PM HD - underdevelopment of ovaries, at IR HD embryo death) and for some pairs of lines are reproduced with $100 \%$ probability. An obligatory condition of the PM HD is the progeny cultivation at an elevated temperature; however, the activity of TEs is affected by temperature only when it causes by severe heat shock incompatible with survival of Drosophila. The degree of dysgenic symptoms is only weakly associated with the number of 
$P$ elements. The presence of a full-sized $P$ element in the genome does not guarantee the induction of HD: the overwhelming majority of natural lines contain it, but the Harwich reference line is usually used as a HD inductor. The contribution of TEs in HD induction may be connected with the change in the conformation of chromatin adjacent to its implantation site and, respectively, due to a change in the regulation of adjacent genes activity. However, taking into account the fact that the places of introduction of the TEs are usually random, and the type of HD manifestations are the same, further investigation of characteristics of the reference for HD lines is required. Hybrid dysgenesis has such serious developmental consequences that it cannot be explained by transposon activation alone. So, the true cause of the dysgenic events has yet to be clarified using modern methods.

Key words: hybrid dysgenesis; transposable elements; transposition rate; Drosophila melanogaster.

For citation: Zakharenko L.P. Doubts about the involvement of transposable elements in the induction of hybrid dysgenesis in Drosophila melanogaster. Pisma v Vavilovskii Zhurnal Genetiki i Selektsii = Letters to Vavilov Journal of Genetics and Breeding. 2021;7(2):109-114. DOI 10.18699/ LettersVJ2021-7-12 (in Russian)

\section{Возникновение гипотезы о причастности} мобильных элементов к индукции гибридного дисгенеза

Синдром внутривидового гибридного дисгенеза (ГД) у дрозофилы, наблюдаемый при скрещивании некоторых пар линий только в одном направлении скрещивания и проявляющий неменделевский характер наследования, известен с 1970-х гг. Две группы исследователей на разных линиях Drosophila melanogaster описали два независимых типа ГД. Один из них назвали РМ ГД, поскольку индуцируется отцовским (Paternal) фактором, взаимодействующим с материнской (Maternal) цитоплазмой (Kidwell et al., 1977), другой - IR ГД (Inducer-Reactive) (Picard, Pelisson, 1979). В это же время в геноме дрозофилы обнаружили мобильные генетические элементы (Ananiev et al., 1978), которые подходили на роль индуктора ГД, поскольку диспергированы по геному, - этим можно было объяснить неменделевский характер наследования синдрома ГД. Кроме этого, перемещаясь, мобильные генетические элементы (МГЭ) потенциально способны индуцировать с некоторой частотой спектр генетических аномалий, сопровождающих синдром ГД (мутации за счет внедрения мобильных элементов в соответствующие гены, нерасхождение хромосом как результат рекомбинаций между МГЭ). Версия о причастности МГЭ к индукции ГД подкреплялась обнаружением независимо возникших видимых мутаций в потомстве от скрещивания референсных линий. В случае РМ ГД в четырех из семи независимых мутантов в гене white обнаружены встройки последовательностей, различающихся по длине в диапазоне 0.5-1.4 Кб (O'Hare, Rubin, 1983). Эти последовательности оказались дефектными производными мобильного элемента. Авторы предположили, что это дефектные производные Р-фактора и по аналогии с Р-фактором назвали данный мобильный элемент Р-элементом. Из девяти видимых мутаций, обнаруженных у дисгенных самок при IR ГД, в одном случае совпадали локализации I-фактора и I-элемента (Pélisson, 1979). Таким образом, название МгЭ образовано от наименований типов ГД. Однако І-элемент и І-фактор или Р-элемент и Р-фактор - это не одно и то же.

Менее известен так называемый HE (Hobo-Empty) ГД, в котором индуктором считается hobo-транспозон (Yannopoulos et al., 1987). Однако однозначной связи между количеством мобильных элементов $P$ и hobo и степенью выраженности симптомов ГД не обнаружено (Onder, Kasap, 2014;
Kozeretska et al., 2016; Bergman et al., 2017; Srivastav, Kelleher, 2017). Более того, скрещивание самцов из линий с большим количеством hobo-элементов с самками, не содержащими элемент hobo, показало, что полученные гибриды более плодовиты, чем их родители (Zakharenko, Perepelkina, 2009).

\section{Р-фактор и Р-элемент - разные понятия}

Распространенность Р- или І-фактора в популяции нельзя автоматически считать распространенностью $P$ - или І-элемента. Необходимо отметить, что в работах прошлых лет, на которые сейчас ссылаются как на доказательство индукции ГД мобильными элементами, версия о причастности МГЭ к ГД сформулирована очень осторожно - как потенциальная возможность такой связи (Pélisson, 1979; Seleme et al., 1999).

Дополнительным аргументом в пользу МгЭ как индуктора ГД послужил тот факт, что в референсной І-линии нашли полноразмерный I-элемент, а в R-линии - только дефектные копии (Busseau et al., 1994). При РМ ГД Р-элементы обнаружены в референсной отцовской линии из природы (Harwich), при этом в материнской лабораторной линии (Canton-S) их не было (O'Hare, Rubin, 1983). Позднее оказалось, что полноразмерные $Р$-элементы содержатся практически во всех линиях из природы, однако только редкие линии могут индуцировать ГД (Itoh et al., 1999, 2001, 2004) и не все линии без Р-элемента отвечают на индукцию (Ignatenko et al., 2015). Несоответствие между цитотипом и наличием/отсутствием МГЭ в линиях стали объяснять тем, что в одних линиях есть репрессоры в виде малых РНК, а в других они отсутствуют.

\section{Малые РНК и их влияние на активность МГЭ}

Стандартное объяснение ГД состоит в том, что яйцеклетки материнских линий не имеют ріРНК к соответствующим МГЭ, которые, предположительно, индуцируют ГД. Однако на примере $D$. virilis показано, что устойчивость к ГД не может быть объяснена материнским пулом ріPHK (Funikov et al., 2018).

По другим данным, ріРНК, которая должна регулировать активность МГЭ, не влияет на уровень транскрипции Р-элемента в ходе РМ ГД (Teixeira et al., 2017). Предполагается, что разница в процессинге $Р$-элемента в соматических и генеративных тканях обусловлена конформацией ДНК. Как это может сказаться на скорости перемещения Р-элемента, в работе не исследовано. 
Связь между количеством ріРНК и делением линий на цитотипы во время РМ ГД обнаружена К.T. Wakisaka и коллегами (2017). По данным авторов, линии с материнским цитотипом $\mathrm{M}^{\prime}$ содержали в геноме Р-элемент и проявили низкий уровень индукционности при скрещивании с самцами линии Harwich (13-28\% дисгенных мyх F1) по сравнению со скрещиванием Canton-S/Harwich (100\% дисгенных мух F1). Однако соотношение ріРНК/Р-элемент в родительских линиях не изучено. Важно отметить, что потомки разных линий, одинаково индифферентных к индукции ГД (так называемые Q-линии), разделились на два класса и имели достоверно различающийся уровень ріРНК/Р-элемент как в овариолах, так и эмбрионах F1 от скрещивания с самцами Harwich (Wakisaka et al., 2017).

Корреляция между реактивностью линий в системе IR ГД и уровнем ріРНК обнаружена у D. melanogaster (Ryazansky et al., 2017). Чем больше ріРНК, по современным представлениям блокирующей активность соответствующего мобильного элемента, тем меньше реактивность линии, измеренная по доле стерильных самок в потомстве от дисгенного скрещивания. Чем меньше реактивность линии, тем ниже должна быть скорость перемещения l-элемента, предположительно вызывающего IR-гибридный дисгенез. Однако скорость перемещения I-элемента в данной работе не исследуется, сообщается лишь, что R-линии с разной реактивностью содержат одинаковое количество копий дефектного l-элемента. Активность (скорость перемещения) l-элемента, таким образом, оценивается косвенно, по проценту стерильных дисгенных самок.

Если активность МГЭ регулируется малыми РНК, то на скорость перемещения МГЭ влияет не столько уровень транскрипции МГЭ, сколько количество малых РНК или соотношение этих показателей. В ооцитах недисгенных самок при IR ГД ріРНК I-элемента накапливается больше, чем в ооцитах дисгенных самок, однако, несмотря на это, в овариолах недисгенных самок транспозаза тоже синтезируется, но накапливается не в ооцитах, а питающих клетках (Brennecke et al., 2008). То есть в потомстве, полученном от межлинейных скрещиваний в разных направлениях, транспорт этого белка из питающих клеток в ооцит различается. Другие белки, влияющие на фертильность гибридного потомства, тоже могут транспортироваться по-разному в клетках потомства, полученного от скрещиваний в разных направлениях. Например, у дисгенных самок D. virilis нарушена миграция стволовых генеративных клеток (Sokolova et al., 2013): нет корреляции между распространением Р-фактора (оценивается по стерильности самок F1 от скрещивания тестируемых линий с референсными линиями) и Р-элемента (оценивается молекулярными методами) (Ignatenko et al., 2015).

Во всех вышеперечисленных работах транспозиционную активность МГЭ, предположительно ответственных за ГД, оценивали косвенно, по проценту самок с дисгенными яичниками.

\section{Скорость перемещения МГЭ при ГД}

Прямое измерение скорости перемещения МГЭ в геноме клеток дисгенных яичников практически невозможно, поскольку яичники гибридных самок недоразвиты. Такие сам- ки не дают потомства, в котором можно было бы оценить частоту перемещения МГЭ в генеративных клетках родителей. Одно перемещение $P$-элемента на Х-хромосому за поколение обнаружено с использованием косвенного подхода у самцов F2, полученных в результате дисгенных скрещиваний (Eggleston et al., 1988). Использование метода полногеномного секвенирования нового поколения дает более высокие оценки скорости перемещения Р-элемента при РМ ГД (Khurana et al., 2011), однако в этой работе ДНК для анализа выделяли из дисгенных недоразвитых яичников, лишенных генеративных клеток, то есть активность $P$-элемента фактически оценивали в соматических клетках. Кроме того, результаты анализа ДНК дисгенных яичников сравнивали не с реципрокным скрещиванием, а родителями, хотя известно, что гибридизация сама по себе может влиять на скорость перемещения МГЭ за счет увеличения числа рекомбинаций между ними (Коваленко и др., 2006).

Таким образом, высокую активность МГЭ постулируют, но истинную скорость перемещения МГЭ при ГД фактически никто не знает. Стоит отметить, при IR ГД часть потомков плодовита, однако заметного увеличения частоты движений l-элемента у них не обнаружили. Скорость перемещения l-элемента при IR ГД составляет около 10(-3) на геном за поколение или по крайней мере на порядок меньше, чем одно перемещение на геном (Jensen, Heidmann, 1991; Pelisson et al., 1991; Busseau et al., 1998), что сопоставимо со спонтанной скоростью движения МГЭ (Charlesworth et al., 1992). Даже генетические конструкции на основе l-элемента под хит-шоковым промотором при IR ГД перемещаются всего лишь с частотой 0.5 на геном за поколение, то есть движение l-элемента наблюдается не в каждом геноме (Seleme et al., 1999).

О скорости перемещения МГЭ при ГД судят, как правило, используя косвенные критерии: уровень нестабильности видимых мутаций, опосредованных внедрением МГЭ (Зайнуллин, Юшкова, 2012); уровень транскрипции транспозазы и количество соответствующих малых регуляторных РНК (Erwin et al., 2015) или чаще всего степень недоразвития яичников исходя из предположения, ставшего со временем догмой, что ГД индуцируется I- или Р-элементом.

Насколько правомочны такие косвенные оценки? Нестабильность видимых мутаций чаще связана с рекомбинациями между МГЭ, а не их перемещением (Eggleston et al., 1988, 1996; Zakharenko et al., 2004). Также без объяснения остается тот факт, что l-элемент, перемещающийся в изогенной линии у cn bw sp без скрещиваний с другими линиями, не индуцирует при этом симптомов ГД (Moschetti et al., 2010), а Р-элемент перемещается в геноме М-линии, при этом цитотип линии не меняется (Biémont, 1994).

\section{Редкие транспозиции МГЭ в различные гены} и высокая воспроизводимость симптомов ГД К настоящему времени известно, что мобильные элементы занимают значительную часть генома дрозофилы и по большей части нейтральны, поскольку локализованы преимущественно в межгенных пространствах и мало влияют на жизнедеятельность (Kapitonov, Jurka, 2003). Особи одной и той же популяции с одинаковыми характеристиками уни- 
кальны по паттерну распределения МГЭ (Charlesworth et al., 1992). Несмотря на наличие горячих сайтов внедрения (Spradling et al., 2011) и то, что транспозаза Р-элемента сайтспецифична и распознает консенсус из 8 пар оснований (O'Hare, Rubin, 1983), Р-элементы могут внедряться практически во все локусы, где есть этот октануклеотид (Cooley et al., 1988). По крайней мере $40 \%$ генов D. melanogaster удалось «испортить» внедрением вектора на базе Р-элемента (Bellen et al., 2004).

При низкой частоте перемещений МГЭ (менее одного на геном за поколение) в непредсказуемые сайты всякий раз индуцируются одни и те же физиологические изменения, строго характерные для разных типов ГД. В одном случае скрещивание реактивных R-самок с индукторными I-самцами референсных линий D. melanogaster приводит к появлению частичной стерильности самок (Picard, 1976), в другом случае (РМ ГД) стерильность характерна для обоих полов (Kidwell et al., 1977; Dorogova et al., 2017). При IR ГД доля стерильных самок уменьшается с возрастом, но у старых самок есть зависимость от температуры содержания. При РМ ГД отмечена зависимость от температуры, но нет зависимости от возраста. При РМ ГД у самок наблюдается недоразвитие яичников за счет элиминации генеративных половых клеток (Dorogova et al., 2017), при IR ГД гибридные самки откладывают яйца, но из них развиваются эмбрионы, погибающие на ранних стадиях развития (Brennecke et al., 2008). Скрещивание референсной для РМ ГД Р-линии с референсной для IR ГД R-линией дает комбинацию симптомов, характерных для этих типов ГД: яичники недоразвиты как при РМ ГД, но нет зависимости от температуры для молодых самок, при этом выявлена связь с возрастом самок как при IR ГД (Khurana et al., 2011). Есть предположение, что РМ ГД может индуцироваться перемещением не одного, а многих МГЭ (Khurana et al., 2011), однако причастность других МГЭ к индукции РМ ГД опровергается исследованиями (Eggleston et al., 1988; Woodruff et al., 1987). Кроме этого, в работах, посвященных изучению природы ГД, всегда сообщают о РМ, IR или HE ГД, а следовательно, о конкретных P-, l- или hoboэлементах соответственно.

\section{Влияние температуры на перемещение МГЭ}

Почему РМ ГД зависит от температуры, неизвестно. Активность по крайней мере 11 МГЭ не меняется при температуре $28^{\circ} \mathrm{C}$, необходимой для индукции ГД (Alonso-González et al., 2006; Vázquez et al., 2007). Изменения в скорости перемещения МГЭ 412 на два порядка по сравнению с контролем до 10(-2) на сайт на геном за поколение или не более одного перемещения на геном за поколение наблюдали лишь при кратковременном тяжелом тепловом шоке $\left(37^{\circ} \mathrm{C}\right)$ (Колесникова и др., 1991; Ratner et al., 1992).

Между тем изменение температурного режима может влиять на множество ферментативных процессов, которые, в частности, могут приводить к недоразвитию гонад в одном направлении скрещивания за счет материнских эпигенетических эффектов, поскольку белки теплового шока играют ведущую роль в реакции клетки на стресс и контроле качества (Morozov et al., 2017).

\section{Заключение}

Гипотеза о причастности массовых перемещений МГЭ к ГД, предложенная полвека назад, казалась логичной и сыграла заметную роль в развитии генной инженерии благодаря использованию векторов, созданных на основе мобильных элементов, и исследовании характеристик самих мобильных элементов. Однако возникают сомнения, насколько хорошо обоснована концепция, согласно которой транспозиции МГЭ ответственны за ГД. Если референсные для ГД линии дрозофил используют для изучения регуляции активности МГЭ, то это не значит, что изменение количества транскриптов МГЭ приводит к смене числа их перемещений.

Возникает вопрос, не является ли синдром ГД прерогативой ограниченного числа референсных линий, различающихся по некоторым параметрам, дисрегулирующим взаимодействие материнской цитоплазмы с отцовским геномом. Причины гибридного дисгенеза для каждой пары линий, скорее всего, уникальны и не связаны с перемещением мобильных элементов. Логично предположить, что линии, содержащие и не содержащие тот или иной мобильный элемент, могут генетически заметно различаться и вследствие значительной генетической дистанции между родителями у гибридного потомства могут возникать симптомы ГД. В природном генофонде $D$. melanogaster существует значительный запас скрытой несовместимости, то есть внутривидовой антагонистический эпистаз у дрозофилы - явление обычное (Corbett-Detig et al., 2013).

Причину ГД, на наш взгляд, необходимо искать в генетической гетерогенности скрещиваемых линий: в первую очередь, важен анализ геномов и транскриптомов референсных линий.

В последние годы систему ГД активно используют для оценки регуляции активности МГЭ у дисгенных гибридов, полученных от скрещивания референсных линий. Вероятно, факторы невыясненной природы влияют не только на проявление симптомов ГД, но и активность МГЭ, особенно если исследование уровня транскрипции и трансляции МГЭ сопровождается измерением скорости их транспозиций. Так, по крайней мере у транспозазы $Р$-элемента пока не обнаружено другой функции кроме вырезания или встраивания.

Гибридный дисгенез значительно влияет на развитие, поэтому его невозможно объяснить только активацией транспозонов. Истинную причину дисгенных событий еще предстоит выяснить с использованием современных методов (Malone et al., 2015).

\section{Список литературы / References}

Зайнуллин В.Г., Юшкова Е.А. Оценка радиационно-индуцированного уровня транспозиций $Р$-элементов в экспериментальных популяциях и лабораторных линиях Drosophila melanogaster. Генетика. 2012;48:561-565.

[Zainullin V.G., lushkova E.A. Estimation of the levels of radiationinduced $P$-element transposition in Drosophila melanogaster experimental populations and laboratory strains. Genetika $=$ Russ. J. Genet. 2012;48:561-565. (in Russian)]

Захаренко Л.П., Захаров И.К., Волошина М.А., Романова О.М., Алексеенко А.А., Георгиев П.Г. Причина сохранения высокой нестабильности по гену yellow в линиях Drosophila melanogaster, выделенных в период «моды на мутацию» в популяции Умани. Генетика. 2004;40:316-321. 
[Zakharenko L.P., Zakharov I.K., Voloshina M.A., Romanova O.M., Alek seenko A.A., Georgiev P.G. Cause for maintaining high instability at the yellow gene in Drosophila melanogaster lines, isolated during the "mode for mutation" period in Uman populations. Genetika = Russ. J. Genet. 2004;40:316-321. (in Russian)]

Колесникова О.В., Забанов С.А., Васильева Л.А., Ратнер В.А. Индукция транспозиций МГЭ Dm-412 в геноме дрозофилы при помощи теплового шока. Генетика. 1991;27:1547-1555.

[Kolesnikova O.V., Zabanov S.A., Vasil'eva L.A., Ratner V.A. Transposition induction of the mobile genetic element Dm412 in the Drosoph ila genome using heat shock. Genetika = Russ. J. Genet. 1991;27:15471555. (in Russian)]

Коваленко Л.В., Захаренко Л.П., Волошина М.А., Карамышева Т.В., Рубцов Н.Б., Захаров И.К. Поведение транспозонов hobo и $P$ в нестабильной линии yellow-2-717 Drosophila melanogaster и ее производных после скрещивания с лабораторной линией. Генетика. 2006;42:748-756.

[Kovalenko L.V., Zakharenko L.P., Voloshina M.A., Karamysheva T.V., Rubtsov N.B., Zakharov I.K. Behavior of hobo and $P$ transposons in yellow2-717 unstable line of Drosophila melanogaster and its derivatives after crossing with a laboratory strain. Genetika = Russ. J. Genet. 2006;42:748-756. (in Russian)]

Alonso-González L., Domínguez A., Albornoz J. Direct determination of the influence of extreme temperature on transposition and structural mutation rates of Drosophila melanogaster mobile elements. Genetica. 2006;128:11-19. DOI 10.1007/s10709-005-2480-6.

Ananiev E.V., Gvozdev V.A., Ilyin Yu.V., Tchurikov N.A., Georgiev G.P. Reiterated genes with varying location in intercalary heterochromatin regions of Drosophila melanogaster polytene chromosomes. Chromosoma. 1978;70:1-17. DOI 10.1007/BF00292211.

Bellen H.J., Levis R.W., Liao G., He Y., Carlson J.W., Tsang G., Evans-Holm M., Robin H.P., Schulze K.L., Rubin G.M., Hoskins R.A., Spradling A.C. The $B D G P$ gene disruption project: single transposon insertions associated with $40 \%$ of Drosophila genes. 2004;167(2):761-81. DOI 10.1534/ genetics.104.026427.

Bergman C.M., Nelson M.G., Bondarenko V., Kozeretska I.A. Genomic analysis of $P$ elements in natural populations of Drosophila melanogaster. PeerJ. 2017;5:e3824. DOI 10.7717/peerj.3824.

Biémont C. Dynamic equilibrium between insertion and excision of $P$ elements in highly inbred lines from an $\mathrm{M}^{\prime}$ strain of Drosophila melanogaster. J. Mol. Evol. 1994;39:466-472. DOI 10.1007/BF00173415.

Busseau I., Chaboissier M.C., Pélisson A., Bucheton A. I factors in Drosophila melanogaster: transposition under control. Genetica. 1994;93:101-116. DOI 10.1007/BF01435243.

Busseau I., Malinsky S., Balakireva M., Chaboissier M.C., Teninges D., Bucheton A. A genetically marked I element in Drosophila melanogaster can be mobilized when ORF2 is provided in trans. Genetics. 1998; 148:267-275.

Brennecke J., Malone C.D., Aravin A.A., Sachidanandam R., Stark A., Hannon G.J. An epigenetic role for maternally inherited piRNAs in transposon silencing. Science. 2008;322:1387-1392. DOI 10.1126/science.1165171.

Charlesworth B., Lapid A., Canada D. The distribution of transposable elements within and between chromosomes in a population of Drosophila melanogaster. I element frequencies and distribution. Genet. Res. 1992;60:103-114. DOI 10.1017/s0016672300030792.

Cooley L., Kelley R., Spradling A. Insertional mutagenesis of the Drosophila genome with single $P$ elements. Science. 1988;239:1121-1128.

Corbett-Detig R.B., Zhou J., Clark A.G. Hartl D.L., Ayroles J.F. Genetic incompatibilities are widespread within species. Nature. 2013;504:135 137. DOI 10.1038/nature12678.

Dorogova N.V., Bolobolova E.U., Zakharenko L.P. Cellular aspects of gonadal atrophy in Drosophila P-M hybrid dysgenesis. Dev. Biol. 2017;424:105-112. DOI 10.1016/j.ydbio.2017.02.020.

Eggleston W.B., Johnson-Schlitz D.M., Engels W.R. P-M hybrid dysgenesis does not mobilize other transposable element families in $D$. me lanogaster. Nature. 1988;331:368-370.

Eggleston W.B., Rim N.R., Lim J.K. Molecular characterization of hobo-mediated inversions in Drosophila melanogaster. Genetics. 1996;144(2):647-656.

Erwin A.A., Galdos M.A., Wickersheim M.L., Harrison C.C., Marr K.D., Colicchio J.M., Blumenstiel J.P. piRNAs are associated with diverse trans- generational effects on gene and transposon expression in a hybrid dysgenic syndrome of D. virilis. PLoS Genet. 2015;11(8):e1005332. DOI 10.1371/journal.pgen.

Funikov S.Y., Kulikova D.A., Krasnov G.S., Rezvykh A.P., Chuvakova L.N., Shostak N.G., Zelentsova E.S., Blumenstiel J.P., Evgen'ev M.B. Spontaneous gain of susceptibility suggests a novel mechanism of resistance to hybrid dysgenesis in Drosophila virilis. PLoS Genet. 2018;14(5):e1007400. DOI 10.1371/journal.pgen.1007400.

Ignatenko O.M., Zakharenko L.P., Dorogova N.V., Fedorova S.A. P elements and the determinants of hybrid dysgenesis have different dynamics of propagation in Drosophila melanogaster populations. Genetica. 2015;143:751-759. DOI 10.1007/s10709-015-9872-z.

Itoh M., Fukui T., Kitamura M., Uenoyama T., Watada M., Yamaguchi M. Phenotypic stability of the P-M system in wild populations of Drosophila melanogaster. Genes. Genet. Syst. 2004;79:9-18. DOI 10.1266/ ggs.79.9.

Itoh M., Sasai N., Inoue Y., Watada M. P elements and P-M characteristics in natural populations of Drosophila melanogaster in the southernmost islands of Japan and in Taiwan. Heredity (Edinb.). 2001;86(Pt 2): 206-212. DOI 10.1046/j.1365-2540.2001.00817.x.

Itoh M., Woodruff R.C., Leone M.A., Boussy I.A. Genomic $P$ elements and P-M characteristics of eastern Australian populations of Drosophila melanogaster. Genetica. 1999;106:231-245. DOI 10.1023/a:1003918417012.

Jensen S., Heidmann T. An indicator gene for detection of germline retrotransposition in transgenic Drosophila demonstrates RNA-mediated transposition of the LINE / element. EMBO J. 1991;10:1927-1937.

Kapitonov V.V., Jurka J. Molecular paleontology of transposable elements in the Drosophila melanogaster genome. Proc. Natl. Acad. Sci. USA. 2003;100:6569-6574. DOI 10.1073/pnas.0732024100.

Kidwell M.G., Kidwell J.F., Sved J.A. Hybrid dysgenesis in Drosophila melanogaster: A syndrome of aberrant traits including mutation, sterility and male recombination. Genetics. 1977;86:813-833.

Khurana J.S., Wang J., Xu J., Koppetsch B.S., Thomson T.C., Nowosielska A., Li C., Zamore P.D., Weng Z., Theurkauf W.E. Adaptation to $P$ element transposon invasion in Drosophila melanogaster. Cell. 2011;147:1551-1563. DOI 10.1016/j.cell.2011.11.042.

Kozeretska I., Bondarenko V., Shulga V., Serga S., Rozhok A., Protsenko O., Nelson M.G. Phenotypic and genomic analysis of $P$ elements in natural populations of Drosophila melanogaster. Mobile DNA. 2016. DOI 10.1101/047910.

Malone C.D., Lehmann R., Teixeira F.K. The cellular basis of hybrid dysgenesis and Stellate regulation in Drosophila. Curr. Opin. Genet. Dev. 2015;34:88-94. DOI 10.1016/j.gde.2015.09.003.

Morozov A.V., Astakhova T.M., Garbuz D.G., Krasnov G.S., Bobkova N.V. Zatsepina O.G., Karpov V.L., Evgen'ev M.B. Interplay between recombinant $\mathrm{Hsp} 70$ and proteasomes: proteasome activity modulation and ubiquitin-independent cleavage of Hsp70. Cell Stress Chaperones. 2017;22:687-697. DOI 10.1007/s12192-017-0792-y.

Moschetti R., Dimitri P., Caizzi R., Junakovic N. Genomic instability of l elements of Drosophila melanogaster in absence of dysgenic crosses. PLoS One. 2010;5:e13142. DOI 10.1371/journal.pone.0013142.

O'Hare K., Rubin G.M. Structures of $P$ transposable elements and their sites of insertion and excision in the Drosophila melanogaster genome. Cell. 1983;34:25-35. DOI 10.1016/0092-8674(83)90133-2.

Onder B.S., Kasap O.E. P Element Activity and Molecular Structure in Drosophila melanogaster Populations from Firtina Valley, Turkey. J. Insect. Sci. 2014;14:16. DOI 10.1093/jis/14.1.16.

Pélisson A. The I-R system of hybrid dysgenesis in Drosophila melanogaster: influence on SF females sterility of their inducer and reactive paternal chromosomes. Heredity (Edinb.). 1979;43:423-428. DOI 10.1038/hdy.1979.92.

Pelisson A., Finnegan D.J., Bucheton A. Evidence for retrotransposition of the I factor, a LINE element of Drosophila melanogaster. Proc. Natl. Acad. Sci. USA. 1991;88:4907-4910. DOI 10.1073/pnas.88.11.4907.

Picard G. Non-mendelian female sterility in Drosophila melanogaster. Hereditary transmission of I factor. Genetics. 1976;83:107-123.

Picard G., Pelisson A. Non-Mendelian female sterility in Drosophila melanogaster: characterization of the noninducer chromosomes of inducer strains. Genetics. 1979;91:473-489.

Ratner V.A., Zabanov S.A., Kolesnikova O.V., Vasilyeva L.A. Induction of the mobile genetic element $D m-412$ transpositions in the Dro- 
sophila genome by heat shock treatment. Proc. Natl. Acad. Sci. USA. 1992;89:5650-5654. DOI 10.1073/pnas.89.12.5650.

Ryazansky S., Radion E., Mironova A., Akulenko N., Abramov Y., Morgunova V., Kordyukova M.Y., Olovnikov I., Kalmykova A. Natural variation of piRNA expression affects immunity to transposable elements. PLoS Genet. 2017;13(4):e1006731. DOI 10.1371/journal. pgen.1006731.

Seleme M.C., Busseau I., Malinsky S., Bucheton A., Teninges D. Highfrequency retrotransposition of a marked I factor in Drosophila melanogaster correlates with a dynamic expression pattern of the ORF1 protein in the cytoplasm of oocytes. Genetics. 1999;151:761-771.

Sokolova M.I., Zelentsova E.S., Shostak N.G., Rozhkov N.V., Evgen'ev M.B. Ontogenetic consequences of dysgenic crosses in Drosophila virilis. Int. J. Dev. Biol. 2013;57:731-749. DOI 10.1387/ijdb.120189me.

Spradling A.C., Bellen H.J., Hoskins R.A. Drosophila $P$ elements preferentially transpose to replication origins. Proc. Natl. Acad. Sci. USA. 2011;108:15948-15953. DOI 10.1073/pnas.1112960108.

Srivastav S.P., Kelleher E.S. Paternal induction of hybrid dysgenesis in Drosophila melanogaster is weakly correlated with both P-element and hobo element dosage. G3 (Bethesda). 2017;7:1487-1497. DOI 10.1534/g3.117.040634

Teixeira F.K., Okuniewska M., Malone C.D., Coux R.X., Rio D.C., Lehmann R. piRNA-mediated regulation of transposon alternative splicing in the soma and germ line. Nature. 2017;552:268-272. DOI 10.1038/ nature25018.

Vázquez J.F., Albornoz J., Domínguez A. Direct determination of the effects of genotype and extreme temperature on the transposition of roo in long-term mutation accumulation lines of Drosophila melanogaster. Mol. Genet. Genomics. 2007;278:653-664. DOI 10.1007/ s00438-007-0282-5.

Wakisaka K.T., Ichiyanagi K., Ohno S., Itoh M. Diversity of $P$-element piRNA production among $M^{\prime}$ and $Q$ strains and its association with $P-M$ hybrid dysgenesis in Drosophila melanogaster. Mob. DNA. 2017;8:13. DOI 10.1186/s13100-017-0096-x.

Woodruff R.C., Blount J.L., Thompson J.N.Jr. Hybrid dysgenesis in D. melanogaster is not a general release mechanism for DNA transpositions. Science. 1987;237:1206-1218. DOI 10.1126/ science.2820057.

Yannopoulos G., Stamatis N., Monastirioti M., Hatzopoulos P., Louis C. hobo is responsible for the induction of hybrid dysgenesis by strains of Drosophila melanogaster bearing the male recombination factor 23.5MRF. Cell. 1987;49:487-495. DOI 10.1016/00928674(87)90451-x.

Zakharenko L., Perepelkina M. The possible effect of transposons on the Drosophila melanogaster somatic mutation and recombination test. Mutat. Res. 2009;670:1-5. DOI 10.1016/j.mrfmmm.2009.06.015.

Конфликт интересов. Автор заявляет об отсутствии конфликта интересов.

Поступила в редакцию 04.03.2021. После доработки 07.04.2021. Принята к публикации 16.04.2021. 